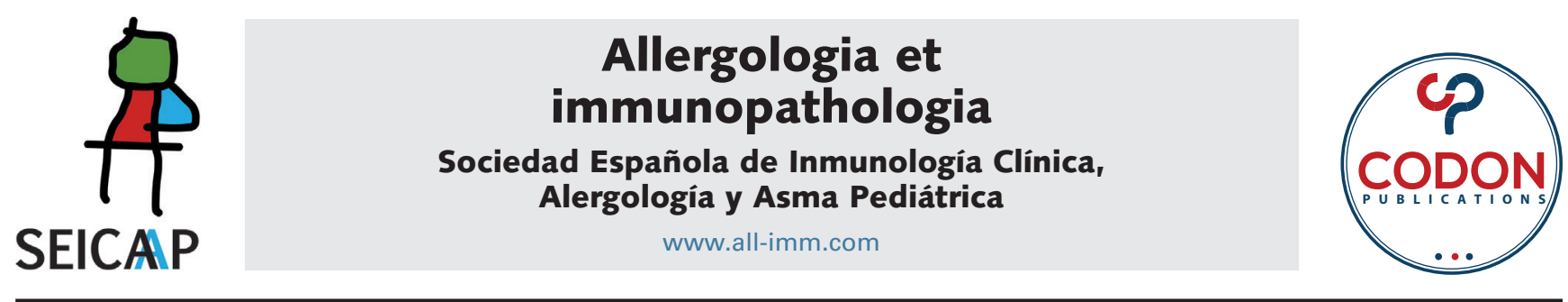

ORIGINAL ARTICLES

OPEN ACCESS (c) $\underset{\mathrm{BY}}{\mathrm{NC}(\mathrm{CA}}$

\title{
Novel BTK mutation in X-linked agammaglobulinemia: Report of a 17-year-old male
}

\author{
Zoha Shaka , Helia Mojtabavi ${ }^{\mathrm{b}, c}$, Elham Rayzan ${ }^{\mathrm{cd}}$, Samaneh Zoghie,f,g,h, \\ Sepideh Shahkarami ${ }^{\text {h,i, }}$, Jimenez Heredia Raule,f,g,k, Iraj Sedighil, Kaan Boztuge,f,g,k,m, \\ Nima Rezaeic, d,h,j*
}

\begin{abstract}
aschool of Medicine, Iran University of Medical Sciences, Tehran, Iran
'School of Medicine, Tehran University of Medical Sciences, Tehran, Iran

'Systematic Review and Meta-Analysis Expert Group (SRMEG), Universal Scientific Education and Research Network (USERN), Tehran, Iran

dInternational Hematology/Oncology of Pediatrics Experts (IHOPE), Universal Scientific Education and Research Network (USERN), Tehran, Iran

eLudwig Boltzmann Institute for Rare and Undiagnosed Diseases, Vienna, Austria

fSt. Anna Children's Cancer Research Institute (CCRI), Vienna, Austria

${ }^{3}$ CeMM Research Center for Molecular Medicine of the Austrian Academy of Sciences, Vienna, Austria

${ }^{h}$ Research Center for Immunodeficiencies, Children's Medical Center, Tehran University of Medical Sciences, Tehran, Iran

'Department of Pediatrics, Dr. Von Hauner Children's Hospital, University Hospital, Ludwig-Maximilians-Universität München (LMU), Munich, Germany

'Medical Genetics Network (Megene), Universal Scientific Education and Research Network (USERN), Tehran, Iran

${ }^{k}$ Department of Pediatrics and Adolescent Medicine, Medical University of Vienna, Vienna, Austria

'Department of Paediatrics, School of Medicine, Hamedan University of Medical Sciences, Hamedan, Iran

${ }^{m}$ Department of Paediatrics and Adolescent Medicine, St Anna Children's Hospital, Medical University of Vienna, Vienna, Austria
\end{abstract}

Received 23 August 2020; Accepted 11 November 2020

Available online 1 March 2021

\section{KEYWORDS}

agammaglobulinemia; immunodeficiency; mutation; tyrosine kinase, X-linked agammaglobulinemia

\begin{abstract}
Introduction and objectives: X-linked agammaglobulinemia (XLA), the first known primary immunodeficiency, is caused by rare mutations in Bruton's tyrosine kinase (BTK) gene. Mutations in the BTK gene lead to a failure in the development and maturation of B-cell linage. A decreased number of B-cells results in agammaglobulinemia and increased susceptibility to a variety of infections. Therefore, patients with XLA usually manifest with repetitive bacterial infections, such as upper respiratory tract infections, septic arthritis, osteomyelitis, and urinary tract infections, since their infancy.

Patients: We report a 17-year-old Iranian boy with XLA, referred to us with a history of severe and recurrent episodes of bacterial infections for a period of six years.
\end{abstract}

*Corresponding author: Nima Rezaei, MD, PhD, Children's Medical Center Hospital, Dr. Qarib St, Keshavarz Blvd, Tehran 14194 , Iran. Email address: rezaei_nima@tums.ac.ir, rezaei_nima@yahoo.com 
Results: Genetic analysis using the whole Exome sequencing revealed a hemizygous missense mutation in the BTK gene (c.428 A > T, p.His143Leu).

Conclusion: To our knowledge, c.428 A > T has not been reported in the BTK gene.

(C) 2021 Codon Publications. Published by Codon Publications.

\section{Introduction}

X-linked agammaglobulinemia (XLA) is an inherited disorder characterized by a profound deficiency of all isotypes of immunoglobulins (Ig) with a significant reduction in mature B-cell counts (less than 1\%) in the peripheral circulation. ${ }^{1}$ This disease belongs to a broader group of rare genetic disorders called primary immunodeficiency diseases (PIDs). $\mathrm{XLA}$, after years of study and gaining a better insight about PIDs, can be described as human inborn errors of immunity. ${ }^{2}$ PID affects different components of innate and adaptive immune system at various levels of development and maturation. Up to date, most reported cases of PID in children are that of XLA as it is known as the most common inherited antibody deficiency. ${ }^{1-4}$

In 1993, Bruton tyrosine kinase (BTK) gene located on X chromosome was found to be the responsible gene defect for XLA. It was named after pediatrician Ogden Bruton, who first discovered the disease in 1952.3 All the blood cell lineages derived from hematopoietic stem cells can express BTK, anon-receptor protein tyrosine kinase, except T-cells and natural killer cells. In B-cell linage, BTK is involved as a key regulator of different signals, transduction pathways, and plays an essential role in the differentiation and maturation of premature B-lymphocytes. Therefore, mutations in the BTK gene result in developmental arrest from the pro-B-cell to pre-B-cell stage. ${ }^{5}$

Owing to the lack of sufficient humoral immunity and failure in the production of immunoglobulins, XLA patients generally suffer from the most severe and recurrent infections. Symptoms typically appear after the age of six months when the protective trans-placental maternal Immunoglobulin G (IgG) antibodies decrease and patients are unable to produce antibodies on their own. ${ }^{4}$ The most common clinical manifestations in XLA patients are upper and lower respiratory tract infections, such as otitis media, sinusitis, bronchitis, and pneumonia. Severe infections, including septicemia, meningitis, osteomyelitis and septic arthritis, and gastrointestinal tract infections, may also occur in XLA patients. ${ }^{2-6}$

Since 1952, immunoglobulin replacement therapy and prophylactic antibiotic therapy are the cornerstone treatments for XLA patients. ${ }^{7}$ In immunoglobulin replacement therapy, a collection of pooled antibodies (>90\% IgG and $<10 \%$ immunoglobulin $A$ [ IgA] and immunoglobulin $M$ $[\operatorname{lgM}])$, obtained from the serum of hundreds of donors, is administered intravenously (IV), subcutaneously (SC), or intramuscularly (IM). ${ }^{8}$ This treatment is usually initiated with a loading dose of $1 \mathrm{~g} / \mathrm{kg}$ body weight and continued lifelong with a maintenance dose of $400-600 \mathrm{mg} / \mathrm{kg}$ body weight every month (IV) or every 15 days (SC). The goal of this treatment is to reduce life-threatening infections and increase the survival rate and quality of life in XLA patients. ${ }^{9}$

\section{Case presentation}

A 17-year-old Iranian boy was referred due to recurrent infections to the Pediatrics Center of Excellence of the Children's Medical Center Hospital, Tehran, Iran. His symptoms started with a fever and chronic productive cough at the age of four, and was hospitalized for recurrent respiratory tract infections such as pneumonia and sinusitis at the age of five. Later, he experienced several episodes of fever and swelling in his right shoulder joint and right knee, which were diagnosed as recurrent osteomyelitis and septic arthritis.

He was the second child of non-consanguineous parents who had lost their first male child at the age of seven because of severe pneumonia. The other two male siblings, aged 15 and 13 years, were asymptomatic at the time of the study. Apart from his brother, in their extended family history, his mother had lost a male sibling in early childhood due to central nervous system (CNS) infection. The other seven siblings of his mother were healthy and totally complaint-free. The family pedigree in detail is illustrated in Figure 1.

Our patient was born with a favorable perinatal and birth history, manifested normal growth indices, and followed through normal developmental milestones. Considering the patient's medical history of severe recurrent infections and family history of deaths of their male relatives at a young age because of infections, immunodeficiency disorders were under consideration. At the age of 13 , an antibody profile study was performed for the patient using the enzyme-linked-immunosorbent serologic assay (ELISA) method, which revealed a total decrease in serum immunoglobulins (serum level of $\operatorname{lgG} 131.1 \mathrm{mg} / \mathrm{dL}$ [range: $667-1464 \mathrm{mg} / \mathrm{dL}$ ] and that of $/ g M 39.1 \mathrm{mg} / \mathrm{dL}$ (range: $49-261 \mathrm{mg} / \mathrm{dL}])$.

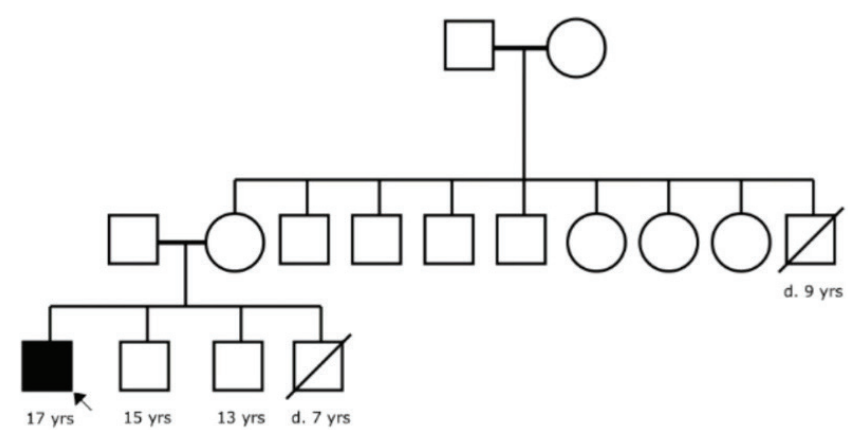

Figure 1 Pedigree of a 17-year-old proband with X-linked agammaglobulinemia. Black square represents affected individuals, the arrow indicates our patient, and members with cross lines are deceased. 
Table 1 Laboratory findings.

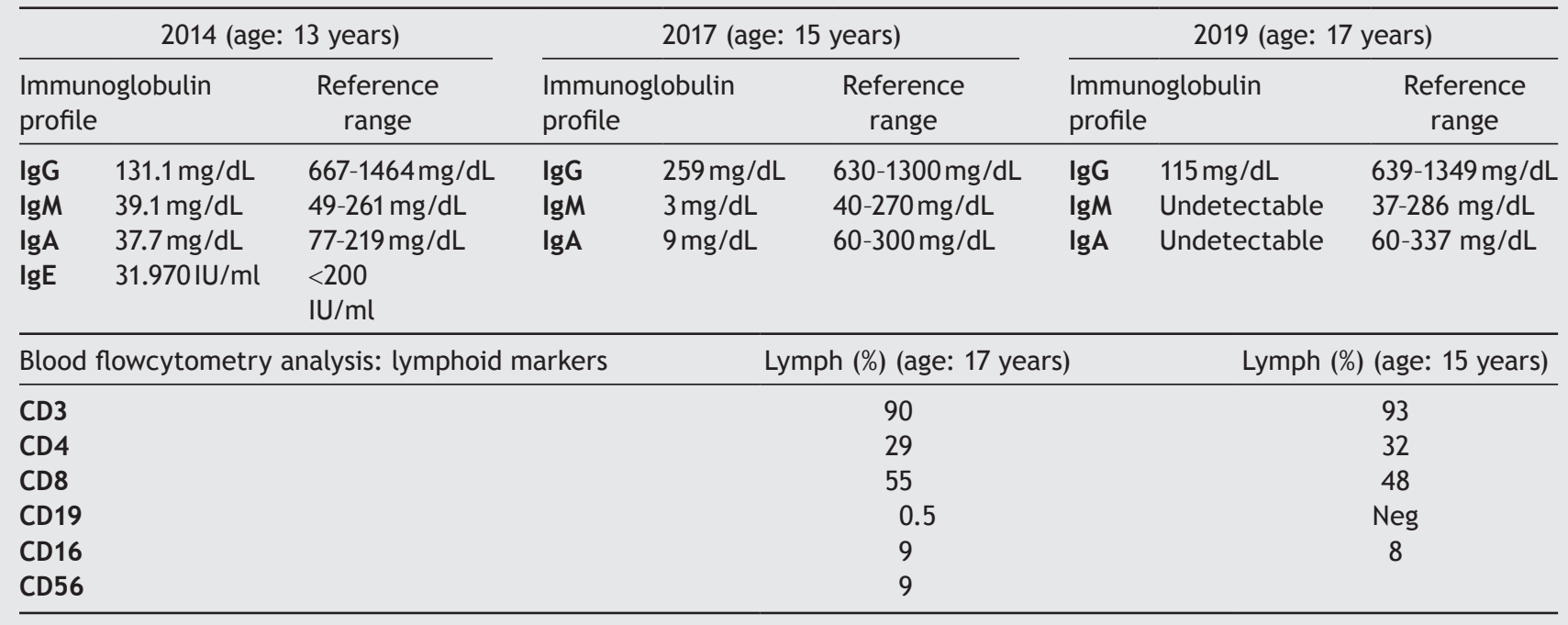

Successively to this result, patient was initiated with intravenous immunoglobulin (IVlg) therapy, $500 \mathrm{mg} / \mathrm{kg}$ body weight stat and a maintenance monthly dose. For the following four years, because of economic burden and limited access to treatment resources, the patient did not adhere to his monthly maintenance therapy. He received just three to four doses in four years. Fortunately, despite discontinuing his treatment and follow-up plan, he did not experience any major episodes of infections since the initial IVIG treatment.

At the age of 17, he was referred to our center for genetic studies. He was symptom-free in between the visits. Another immunological workup, which was performed using the ELISA method, showed a decreased serum level of IgG $115 \mathrm{mg} / \mathrm{dL}$ (range: $639-1349 \mathrm{mg} / \mathrm{dL}$ ) and undetectable levels of IgM and IgA. Detailed levels of initial and successive immunoglobulins are presented in Table 1 . His total leukocytes count was $5.58 \times 10^{3}$ (granulocytes 54\%, monocytes $9 \%$, and lymphocytes $37 \%$ ), which led us to perform flowcytometric study of his blood sample. Flowcytometric analysis was done on 20,000 WBC/ $\mu \mathrm{L}$; it revealed $90 \% \mathrm{CD} 3+$ lymphocytes (in total: 32\%), CD19+ lymphocyte count was $0.5 \%$ (in total: $0.1 \%$ ), and undetectable CD20+.

The result of genetic analysis by whole exome sequencing (WES) confirmed the novel hemizygous missense mutation (ENST00000308731.7:c.428A>T, p.His143Leu) in the BTK gene; hence, XLA diagnosis was confirmed for the patient. The variant was not found in public databases such as 1000 genome project or GnomAD and had a damaging prediction score (v1.4 CADD score of 25.8)..$^{10,11}$

\section{Discussion}

$\mathrm{XLA}$ is a rare disease, so it is difficult to determine the exact number of its prevalence and frequency; however, it has been reported that the prevalence of this disease is around $0.0004 \%$ ( 1 in 250,000) of live births and the disease frequency is approximately $0.001 \%$ ( 1 case in 100,000) in male newborns. ${ }^{6,9}$
XLA could be diagnosed based on the following three main criteria: (1) clinical presentation of recurrent infections in the first five years of life with low levels of immunoglobulins (IgG level less than $500 \mathrm{mg} / \mathrm{dL}$, and IgA and IgM levels less than two standard deviations of the normal range and less than $2 \%$ of mature B-cells in peripheral circulation); (2) a family history of maternal male relatives with XLA diagnosis; and (3) bearing a mutation in BTK gene and/or defective BTK protein. ${ }^{2,12}$

BTK mutations are found in $80 \%$ of patients with agammaglobulinemia. ${ }^{13}$ This gene is located on X-chromosome at Xq21.3-Xq22, consisting of 19 exons and encompasses 37.5 kilo-base $(\mathrm{kb})$ pair of human genome. ${ }^{13}$ More than thousands of different mutations have been found in BTK, with missense mutations being the most frequent ones. In line with this, the mutation that we described in our patient was a novel hemizygous missense mutation (c.428A $>\mathrm{T}$, p.His143Leu).

Even though the genetic analysis is a powerful tool for diagnosing XLA, it cannot determine the prognosis and severity of the disease. In fact, the genotype-phenotype correlation has not been entirely understood yet. Some studies suggest that the severity of XLA disease can be influenced by specific mutations, while several others have concluded that the correlation between genotype and phenotype in XLA is not significant. 6,14,15

The main treatment for this disease is the lifelong immunoglobulin replacement therapy for every three to four weeks, in addition to the use of prophylactic or intensive antibiotic treatment in case of more infections. The IVIG therapy is an effective method to decrease the risk of infection in XLA patients. ${ }^{6,14}$ As a matter of fact, our case did not experience the pre-mentioned infection episodes yet after receiving the IVIG therapy.

Over the past two decades, the prognosis of XLA has improved significantly and patients can survive into the adulthood as the result of early diagnosis of XLA, immunoglobulin replacement therapy, and better management in treating infections. It has been reported in some previous studies that the annual mortality rates of XLA have been 
dropped from $17-25 \%$ to $1 \%$. However, chronic lung diseases and sepsis are still the most common cause of death in these patients, and because of lung complications, more than half of the patients die before reaching the age of 45. ${ }^{5,7,16}$

Even though the immunoglobulin replacement therapy has improved life expectancy and quality of life in XLA patients, it is not a curative and final treatment for XLA and has some limitations. For instance, the high cost of IVIG in most countries often combined with their limited resources, a lifelong course of follow-up, and failing against some pathogens because of the lack of $\lg M$ and $\lg A$ are some of the limitations of this therapy.

Other treatment options for XLA patients are hematopoietic stem cell transplantation (HSCT) and stem cell gene therapy by viral vectors. These treatments are still under study and need to be improved, but the gene therapy has the potential to be the future ideal treatment for XLA, which can cure the defected BTK gene for life..$^{5,7,9}$

\section{Ethical disclosure}

After describing the novelty of genomic mutation causing the disease to the patient's family, they orally consented to the authors to use patient's medical records for publication.

\section{References}

1. Suri D, Rawat A, Singh Surjit. X-linked Agammaglobulinemia. Indian J Pediatr. 2016;83(4):331-7. https://doi.org/10.1007/ s12098-015-2024-8

2. Winkelstein JA, Marino MC, Johnston RB, Jr, Boyle J, Curnutte J, Gallin JI, et al. Chronic granulomatous disease: Report on a national registry of 368 patients. Medicine (Baltimore) 2000;79(3):155-69. https://doi.org/10.1097/00005792-20000500000003

3. Vihinen M, Kwan SP, Lester T, Ochs HD, Resnick I, Väliaho $\mathrm{J}$, et al. Mutations of the human BTK gene coding for bruton tyrosine kinase in $\mathrm{X}$-linked agammaglobulinemia. Hum Mutat. 1999;13(4):280-5. https://doi.org/10.1002/(SICI)10981004(1999)13:4<280::AID-HUMU3>3.0.CO;2-L

4. Kanegane $\mathrm{H}$, Futatani $\mathrm{T}$, Wang $\mathrm{Y}$, Nomura $\mathrm{K}$, Shinozaki K, Matsukura $\mathrm{H}$, et al. Clinical and mutational characteristics of X-linked agammaglobulinemia and its carrier identified by flow cytometric assessment combined with genetic analysis. J Allergy Clin Immunol. 2001;108(6):1012-20. https://doi. org/10.1067/mai.2001.120133
5. Hendriks RW, Bredius RG, Pike-Overzet K, Staal FJJ Eoott. Biology and novel treatment options for XLA, the most common monogenetic immunodeficiency in man. Expert Opin Ther Targets. 2011;15(8):1003-21. https://doi.org/10.1517/1472 8222.2011.585971

6. Lougaris V, Soresina A, Baronio M, Montin D, Martino S, Signa S, et al. Long term follow-up of 168 patients with X-linked agammaglobulinemia reveals increased morbidity and mortality. JAllergy Clin Immunol. 2020 Aug;146(2):429-437 2020. https:// doi.org/10.1016/j.jaci.2020.03.001

7. Shillitoe B, Gennery AJCl. X-linked agammaglobulinaemia: Outcomes in the modern era. Clin Immunol; 2017;183:54-62. https://doi.org/10.1016/j.clim.2017.07.008

8. Pyne D, Ehrenstein M, Morris VJR. The therapeutic uses of intravenous immunoglobulins in autoimmune rheumatic diseases. Rheumatology (Oxford) 2002;41(4):367-74. https://doi. org/10.1093/rheumatology/41.4.367

9. Mazhar M, Waseem M. Agammaglobulinemia. [Internet]. Treasure Island (FL): StatPearls Publishing; 2020 Jan.

10. Karczewski KJ, Francioli LC, Tiao G, Cummings BB, Alföldi J, Wang $\mathrm{Q}$, et al. The mutational constraint spectrum quantified from variation in 141,456 humans. Nature. 2020;581(7809):43443. https://doi.org/10.1530/ey.17.14.3

11. Kircher $M$, Witten DM, Jain P, O'Roak BJ, Cooper GM, Shendure JJ Ng. A general framework for estimating the relative pathogenicity of human genetic variants. Nat Genet. 2014;46(3):310-5. https://doi.org/10.1038/ng.2892

12. Carrillo-Tapia E, García-García E, Herrera-González NE, Yamazaki-Nakashimada MA, Staines-Boone AT, SeguraMendez NH, et al. Delayed diagnosis in X-linked agammaglobulinemia and its relationship to the occurrence of mutations in BTK non-kinase domains. Expert Rev Clin Immunol. 2018;14(1):83-93. https://doi.org/10.1080/17446 66X.2018.1413349

13. Lee J, Rhee M, Min TK, Bang HI, Jang M-A, Kang E-S, et al. A novel BTK gene mutation, c. 82delC (p. Arg28 Alafs* 5), in a Korean family with X-linked agammaglobulinemia. Korean J Pediatr. 2016;59(Suppl 1):S49. https://doi.org/10.3345/ kjp.2016.59.11.S49

14. Han S-P, Lin Y-F, Weng H-Y, Tsai S-F, Fu L-SJFii. A novel BTK gene mutation in a child with atypical $X$-linked agammaglobulinemia and recurrent hemophagocytosis: A case report. Front Immunol. 2019;10:1953. https://doi.org/10.3389/fimmu. 2019.01953

15. Abolhassani H, Vitali M, Lougaris V, Giliani S, Parvaneh N, Parvaneh L, et al. Cohort of Iranian patients with congenital agammaglobulinemia: Mutation analysis and novel gene defects. Expert Rev Clin Immunol. 2016;12(4):479-86. https:// doi.org/10.1586/1744666X.2016.1139451

16. El-Sayed ZA, Abramova I, Aldave JC, Al-Herz W, Bezrodnik L, Boukari R, et al. X-linked agammaglobulinemia (XLA): Phenotype, diagnosis, and therapeutic challenges around the world. World Allergy Organ J. 2019;12(3):100018. https://doi. org/10.1016/j.waojou.2019.100018 